been found to evince any of these effects and analyse the structural, mechanical and thermodynamic features that underly them. These papers are essential reading for anyone who would like to have a go at a solidstate engine. Delaey and his colleagues have provided the steam tables, as it were, of the solid-state engine.

Tong and Wayman have recently published another paper (Acta Met., 23, $209 ; 1975$ ) in which they analyse some of the thermodynamic variables that predispose an alloy to thermoelastic or shape memory behaviour. One interesting conclusion, which is given an interpretation in terms of the 'quasichemical' bond-energy approach, is that an ordered alloy (such as $\mathrm{Fe}_{3} \mathrm{Pt}$ ) has a lower latent heat of transformation, and is more easily able to undergo thermoelastic transformation, than the same alloy in an ordered form. This seems to be a generally valid conclusion.

The solid-state engine may well be able to compete with other physical devices, with or without radiation concentrators, for extracting power from solar radiation. According to Brinkworth (Solar Energy for Man, Compton Press, 1972) such devices have ideal efficiencies in the range $\frac{1}{2}-1 \%$ (for simple thermoelectric generators) to as much as $30 \%$ for thermionic generators with concentrators. The latter are likely to be even more expensive on capital than a solid-state engine, and do not directly generate motion. The engine would seem to deserve some further investigation, which will certainly require the collaboration of metallurgists and mechanical engineers.

\section{Successful quake prediction made}

\section{from Peter J. Smith}

THERE is now ample evidence that some small to moderate earthquakes are immediately preceded by a period during which the seismic velocity ratio $\left(V_{\mathrm{P}} / V_{\mathrm{S}}\right)$ in the vicinity is anomalously low. This effect was first observed by Kondratenko and Nersesov (Tr. Inst. Fiz. Zemli Akad. Nauk SSR, 25, 130; 1962 ) in the Garm region of the USSR for events in the magnitude range 4-5; but similar $V_{\mathrm{P}} / V_{\mathrm{S}}$ changes have since been detected in respect of magnitude 2-3.3 earthquakes at Blue Mountain Lake (New York State), the magnitude 6.4 San Fernando earthquake of 1971, the magnitude 5 Bear Valley (California) earthquake of 1972 , two shallow earthquakes in Japan and several other events in various parts of the world The importance of this phenomenon lies in its potential for earthquake prediction, the more especially as the duration of the precursory $V_{\mathrm{P}} / V_{\mathrm{S}}$ anomaly seems to be related to shock magnitude. But can the potential be converted to practice? In each of the examples cited above, the precursory behaviour was detected by data analysis subsequent to the event. Aggarwal et al. (J. geophys. Res., 80, 718; 1975), however, are now able to report that they have made a genuine and successful prediction.

It was Aggarwal and his colleagues who caried out the original study of the 1971 earthquakes at Blue Mountain Lake, thus becoming the first (Nature, 241, 101; 1973) to confirm the Soviet observations of ten years before. The larger events in the 1971 series were preceded by $V_{\mathrm{P}}$ decreases of $15-20 \%$ and $V_{\mathrm{S}}$ decreases of $5-10 \%$. Subsequently, however, the region became relatively quiet seismically until on 14-15 July 1973 the calm was shattered by two magnitude 3.6 and four rather smaller earthquakes. In response to this renewed activity, Aggarwal et al. immediately (15-16 July) set up a system of portable seismographs and strong motion accelerographs to supplement (for six weeks) the permanent BML station.

During the first third of this period, the recording system indicated an average $S$ wave- $P$ wave travel time ratio $\left(t_{\mathrm{S}} / t_{\mathrm{P}}\right)$ of 1.73 . Over the few days following $30 \mathrm{July}$, however, $t_{\mathrm{s}} / t_{\mathrm{P}} \mathrm{de}-$ creased to about 1.5 . In 1971 the corresponding 'normal' value of $t_{\mathrm{s}} / t_{\mathrm{P}}$ had been about 1.75 and reductions comparable to that now observed had preceded by several days' earthquakes of magnitude 2.5-3.3. Aggarwal and his colleagues thus took the $t_{\mathrm{S}} / t_{\mathrm{P}}$ decrease of 31 July-2 August 1973 to herald a shock of comparable magnitude. On the night of 1 August they therefore predicted that an earthquake of magnitude 2.5-3.0 would occur within a few days.

The maximum magnitude of the apparently impending event was estimated from the inferred spatial extent of the $t_{\mathrm{s}} / t_{\mathrm{P}}$ anomaly. In contrast with the situation in 1971, the most distant (common) station in 1973 remained unaffected and thus presumably lay outside the anomalous zone. Assuming the size of the anomalous region to be directly proportional to the magnitude of the responsible event, and comparing the 1973 data with those of 1971 , the upper magnitude limit of the expected earthquake was about 3 . The minimum magnitude was obtained by estimating the minimum duration of the $t_{\mathrm{s}} / t_{\mathrm{p}}$ anomaly. The 1971 results had showed that, following its initial rapid decrease, $t_{\mathrm{S}} / t_{\mathrm{P}}$ had returned to its normal value rather more slowly and had reached it about one day before the earthquake occurred. As by 1 August $1973 t_{\mathrm{s}} / t_{\mathrm{P}}$ showed no sign of increasing. precedent suggested that the expected earthquake was unlikely to occur for at least two days, making a total $t_{\mathrm{S}} / t_{\mathrm{P}}$ anomaly time of at least four days. And according to the previously established magnitude-precursor time relationship (Scholz et al., Science, 181, 803; 1973), this would correspond to a magnitude of 2.5 .

The earthquake which occurred close to the edge of Blue Mountain Lake on 3 August 1973 had in fact a magnitude of 2.6. Aggarwal and his colleagues are thus the first to predict successfully the place, time and size of an earthquake in the United States and the first to make practical use of precursory seismic velocity changes. Moreover, in their method the precursory interval (whose onset may be detected but whose duration cannot be known until $t_{\mathrm{S}} / t_{\mathrm{P}}$ returns to normal just before the impending event, which may be too late) may be used for predictive purposes before it is complete. If, as seems likely, the size of the anomalous region associated with an impending earthquake is a unique function of the shock magnitude, it follows that once the anomaly appears (and assuming it grows rapidly to its full extent) its spatial dimensions should give magnitude directly. The magnitude can then be fed into the known magnitude-precursor time relationship to determine how long the anomaly will persist and thus just when the earthquake will occur. In short, in any particular case prediction may not have to await the gradual approach of $t_{\mathrm{s}} / t_{\mathrm{p}}$, towards its normal value; valuable time may be gained by making the critical measurements before the rise in $t_{\mathrm{S}} / t_{\mathrm{P}}$ even begins.

Obviously, for this method to succeed it is essential that the size of the anomalous zone be measured reasonably accurately; and here there are difficulties. For one thing, the size of the zone may vary with time, which would complicate the supposed relationship between zone size and magnitude. The temporal characteristics of this relationship are not yet known, although there is evidence that if the anomalous zone grows it must grow relatively rapidly. Second, it would clearly be advantageous to use explosive sources to determine precursory velocity changes and thus define the extent of the anomalous region. But in this case care must be taken to ensure that the waves actually penetrate the zone of anomaly. Aggarwal et al. show that large changes in $V_{\mathrm{p}}$ and $V_{\mathrm{S}}$ are not observed at shallow depths (less than $0.5 \mathrm{~km}$ at BML) for near-horizontal wave paths. This could mean either that significant velocity anomalies do not exist at these depths or that the cracks there are predominantly horizontal. Either way, some sort of anisotropy is implied; and this is likely to complicate the mechanics of the prediction method. 